https://artnodes.uoc.edu

\title{
Hacia la artesanía generativa. Caso de estudio del oficio artesanal del barniz de Pasto (Colombia) y la impresión 3D
}

\author{
Carlos Córdoba-Cely \\ Universidad de Nariño, Colombia \\ Arturo de la Cruz Escobar \\ Universidad de Nariño, Colombia
}

Fecha de presentación: septiembre de 2020

Fecha de aceptación: noviembre de 2020

Fecha de publicación: enero de 2021

\section{Cita recomendada}

Córdoba-Cely, Carlos; Cruz Escobar, Arturo de la. 2021. «Hacia la artesanía generativa. Caso de estudio del oficio artesanal del barniz de Pasto (Colombia) y la impresión 3D». En: Benítez, Laura; Berger, Erich (coord.). «Artes en tiempos de pandemia». Artnodes, núm. 27: 1-9. UOC. [Consulta: dd/mm/aa]. http://doi.org/10.7238/a.v0i27.375112

\section{Resumen}

En la actualidad, se comienza a vislumbrar las implicaciones del nuevo modelo productivo que propone la fabricación digital en el contexto de las prácticas sociales. En el caso particular de la artesanía, no es del todo evidente cómo este tipo de tecnología, que permite materializar cualquier tipo de objeto a partir de bits, contribuye en un determinado sistema de producción cultural. A partir de esta inquietud, y utilizando el concepto de posible adyacente de Stuart Kauffman, se llevó a cabo una experimentación con procesos de impresión 3D y la técnica artesanal del sur de Colombia denominada barniz de Pasto, con el objetivo de explorar los posibles vínculos de estos nuevos modelos productivos con la artesanía. Para justificar la 


\title{
artnodes
}

https://artnodes.uoc.edu

Hacia la artesanía generativa. Caso de estudio del oficio artesanal del barniz de Pasto (Colombia) y la impresión 3D

experimentación en este documento, primero se aborda el concepto de artesanía como sistema híbrido de producción cultural, el cual se modela por la tensión entre los cambios tecnológicos y la conservación del patrimonio simbólico de un colectivo. En segundo lugar, se identifican las características más relevantes que determinan la fabricación digital como posible adyacente de la artesanía en la era posindustrial. Los resultados de la experimentación muestran que la fabricación digital se explica como un vector primario de cambio potencial sobre los oficios artesanales en tres diferentes escenarios posibles: escenario de mayor libertad morfológica, escenario de mayor libertad de manufactura y escenario de mayor libertad creativa. Se propone que estos entornos conjuntos son características de un posible adyacente, al cual se ha denominado artesanía generativa.

\section{Palabras clave}

Artesanía generativa, posible adyacente, fabricación digital, barniz de Pasto, impresión 3D

\section{Towards generative craftwork - Study case of the Pasto varnish (Colombia) artisanal technique and 3D printing}

\begin{abstract}
Currently, we are beginning to envisage the implications of the new productive model which digital manufacturing is offering in the context of social practices. In the particular case of craftwork, it is not entirely clear how this kind of technology, which makes it possible for any kind of object to materialise from bits, contributes in a given system of cultural production. In order to investigate further into this subject, and using the concept of Stuart Kauffman's Adjacent Possible, an experimentation was conducted with 3D printing processes and the artisanal technique from the south of Colombia named Barniz de Pasto (Pasto varnish), with the objective of exploring the possible links that these new productive models have with craftwork. In order to justify the experimentation in this document, firstly the concept of craftwork is addressed as a hybrid system of cultural production, which is modelled by the tension between technological changes and the conservation of the symbolic heritage of a collective. Secondly, the most important characteristics are identified which determine digital manufacturing as an adjacent possible of craftwork in the post-industrial era. The results of the experimentation show that digital manufacturing can be seen to be a primary vector of potential change on artisanal techniques in three different possible settings: the setting of greater morphological freedom, the setting of greater manufacturing freedom and the setting of greater creative freedom. It is proposed that these combined environments are characteristic of an adjacent possible, which has been named Generative Craftwork.
\end{abstract}

\section{Keywords}

generative craftwork, adjacent possible, digital manufacturing, Pasto varnish, 3D printing

\section{Artesanía como sistema híbrido}

La palabra artesanía deriva del término latino artis manus, que significa 'arte con las manos'. Esta base etimológica permite dilucidar la existencia de una aproximación del término desde lo simbólico y lo práctico. El teórico de arte latinoamericano Juan Acha (1979) entiende la artesanía como un «sistema de producción sociocultural» regido por la subjetividad estética. Este sistema puede dividirse en un tipo de artesanía cultural antropológica y un tipo de artesanía cultural productiva. Así, mientras la primera comprende los bienes autóctonos y su producción cultural, la segunda incluye los sistemas de producción basados en innovaciones tecnológicas.

De igual manera, DanielVega (2012) encuentra que existe una artesanía cultural de carácter patrimonial, la cual deviene del reconocimiento del bien cultural dado desde la diversidad; y una artesanía gremial, la cual se origina a principios del siglo xix en la incorporación de diferentes desarro\|los tecnológicos, como el telar mecánico y la máquina de vapor. Según Enrique Gaviria (2002), la reproducción de este conocimiento especializado deriva en organizaciones jerárquicas constituidas por maestros, oficiales y aprendices, donde el colectivo de artesanos se convierte en la primera masa de trabajadores urbanos con cierto sentido de clase del siglo XIX. 


\section{artnodes}

https://artnodes.uoc.edu

Este tipo de artesanía orientada a competir con la línea de producción industrial claramente se aleja del concepto de manufactura realizada a mano, pero no deja de considerarse un oficio artesanal. Por ejemplo, para Gillo Dorfles (1973), el advenimiento de la Revolución Industrial introduce el concepto de estandarización en las artes, con lo cual se da una escisión entre las «artes aplicadas», donde se agrupan un conjunto de oficios manuales como la cerámica, el repujado y los tejidos bordados entre otros, y las «artes puras», donde se encuentran la pintura, la escultura y la música. Para este teórico, la implicación utilitaria de este primer tipo de artes conlleva la introducción parcial de una máquina, con lo cual se afecta la carga simbólica de estos artefactos, que dejan de orientarse al colectivo para pasar a orientarse al mercado. Sin embargo, este tipo de productos tecno-culturales (ya no artefactos) siguen perteneciendo a las artes aplicadas y se pueden denominar como objetos fuera de serie, porque son escogidos por razones afectivas, esnobistas o de diferenciación social (Dorfles 1973: 66).

Parece evidente que la agremiación artesanal ha convertido al artesano en obra de mano calificada mal remunerada, especialmente en aquellos oficios donde el trabajo manual puede llegar a ser reemplazable. Timothy Scrase (2003) considera que este tipo de artesanía de mercado ha dividido la mano de obra artesanal en «élite» y «cotidiana». Así, mientras los primeros realizan productos únicos de nicho hechos a mano con altos estándares de calidad para un público reducido, los segundos elaboran productos seriados terminados a mano con medianos o bajos estándares de calidad para un público masivo.

En este punto, parece que existieran dos visiones opuestas para entender la artesanía como construcción teórica. Sin embargo, es todo lo contrario. La artesanía se constituye como un punto de encuentro híbrido de producción cultural y tecnológica, donde las prácticas subalternas y los sistemas hegemónicos se interpenetran por medio de artefactos manufacturados con la intención de representar el mundo (García Canclini, 1990; Marshall, 2002). Con esta perspectiva orientada desde la hibridación cultural (García Canclini, 1987; Freitag, 2014), la artesanía puede entenderse como un sistema donde diversas prácticas populares y tecnológicas se intersectan para definir los artefactos simbólicos que definen su propio tiempo. Una de las principales ventajas de abordar la artesanía desde este enfoque consiste en comprender la importancia que los artesanos otorgan a la tecnología, más allá de la agremiación de colectivos y la economía de mercado. Los dispositivos tecnológicos modelan de muchas maneras el oficio artesanal, pero, en la mayoría de los casos, es el colectivo artesanal quien incorpora herramientas que extienden la habilidad de sus manos hacia nuevos límites de competencias y experiencias. La artesanía parece funcionar como un entorno de exaptación para dispositivos tecnológicos que permiten la producción y el disfrute de artefactos. Para Gloria Barrera y Cielo Quiñones (2006), la principal característica de la artesanía híbrida consiste en su connotación
Hacia la artesanía generativa. Caso de estudio del oficio artesanal del barniz de Pasto (Colombia) y la impresión 3D

creativa y recreativa, es decir, en su doble condición de ofrecer utilidad y disfrute a un grupo social determinado. Bajo este precepto, se encuentra orientado el concepto de «artesano industrial» que acuña Chris Anderson (2012: 67) y que se verá con detalle a continuación.

\section{Fabricación digital en la era posindustrial}

Según Neil Gershenfeld (2012), el uso de herramientas controladas por computadora tiene sus raíces en 1952 cuando investigadores del MIT conectaron una computadora a una fresadora mecánica para crear el primer aparato de control numérico del mundo. Este dispositivo mixto permitió la digitalización de diferentes procesos de producción y la posibilidad de que los bits se materializaran a través de movimientos de mecanización sustractiva en superficies y volúmenes. A partir de los años ochenta del siglo xx, aparece la fabricación aditiva, donde las herramientas controladas por computador agregan material al proceso, como la impresión 3D, con lo cual los bits adquieren la cualidad directa de transformarse en objetos tridimensionales. De esta manera, el conjunto de herramientas de manufactura aditiva y sustractiva constituyen la primera denominación del término «fabricación digital», entre las cuales se hallan las impresoras 3D que adicionan material por medio de luz ultravioleta (estereolitografía), por fusión térmica (sinterización selectiva por láser) o por medio de deposición de filamento fundido (fused filament fabrication, FFF), así como máquinas de sustracción de materiales tales como cortadoras láser y fresadoras CNC.

Sin embargo, es el mismo Gershenfeld quien asegura que la verdadera revolución tecnológica no está contenida en el tipo de fabricación aditiva o sustractiva, sino en «la posibilidad de convertir los datos en cosas y las cosas en datos» (Gershenfeld, Gershenfeld y Cutcher-Gershenfeld 2017: 44). Es aquí donde la fabricación digital se propone como un cambio paradigmático tecnológico a través de un nuevo sistema de equivalencia y manipulación entre bits y átomos, el cual ha sido denominado como la tercera revolución digital. Así, mientras la primera revolución digital sucede en las comunicaciones con el cambio de redes conmutadas analógicas a protocolos digitales tipo VoIP, la segunda revolución ocurre en la computación con el aumento de la capacidad de procesamiento de los equipos de cómputo para investigación y uso personal. Por último, la actual revolución se desarrolla en el área de la fabricación, con la posibilidad de transformar los bits en átomos y los átomos en bits.

La llegada de la fabricación digital supone una nueva era posindustrial, donde el espíritu distribuido en la producción es una de sus principales características por medio de la recomposición de la dialéctica global-local, al hacer que los bits viajen globalmente mientras que los átomos permanezcan locales (Gershenfeld, Gershenfeld y Cutcher-Gershenfeld 2017:8). En el caso de lo local, 


\section{artnodes}

https://artnodes.uoc.edu

la fabricación digital facilita lo que antes parecía imposible: el acceso a la producción a pequeña escala con costos sostenibles. Es decir, la era posindustrial se caracteriza, ante todo, por la fabricación ubicua y la fabricación a escala humana (pequeña serie) (Merchán, Merchán, Salamanca, Pérez y Nogales 2019; Andersen y Pitkänen 2019). Sobre este punto, Chris Anderson hace un paralelo entre este nuevo sentido de producción a la medida y su teoría de la Cola Larga (Long Tai). Retomando el concepto de bienes de nicho (Anderson 2012:73), este autor explica la existencia de un desplazamiento de la economía moderna hacia un nuevo modelo de mercado para productos de nicho, en contraposición al tradicional mercado genérico para productos masivos. Esta pequeña producción tecnificada es similar a la ocurrida en algunos países como Italia en los años setenta del siglo xx, donde se acuñó el término «producto fuera de serie» y «pequeña serie» para dar a entender la implicación de estándares de fabricación industrial, pero con detalles de personalización manual, similares a la artesanía gremial promovida en la Revolución Industrial (Dorfles 1973:65).

Para algunos investigadores, parece existir una transición de la producción en masa a la producción distribuida de nicho, en una evolución natural económica hacia un mercado centrado en la flexibilidad creativa y la producción a la medida (Atkinson 2004; Piore y Sabel 1999). Como hipótesis de trabajo, se puede asumir que, en la actualidad, la fabricación digital se encuentra abriendo nuevos escenarios de posibilidades para la artesanía, de igual manera como ocurrió a principios del siglo XIX con la estandarización en los procesos de manufactura de diversos oficios populares. Sin embargo, es importante considerar para esta hipótesis una aproximación desde la innovación, la cual vaya más allá del modelo de producción y mercado, y con el cual se justifique el impacto creativo de la fabricación digital en la artesanía. Para esto, es necesario abordar esta tecnología como un componente de innovación inevitable en el ecosistema artesanal, por medio del cual es permitido identificar aquellos escenarios de primer grado donde el sistema ampliará su realidad. Este enfoque es factible desde el concepto de posible adyacente, término acuñado por Stuart Kauffman (1996) para explicar el inevitable y finito crecimiento de cualquier biosfera cada vez que se incrementa su diversidad molecular. Por ejemplo, los ácidos grasos se combinan de tal manera aleatoria que en algún momento forman membranas que separan su estructura interior del ambiente exterior. Este cambio permite un adyacente posible para que estas biomoléculas puedan contener un código genético más seguro 0 mantener mayores reservas de comida. Tal vez lo más interesante de esta idea sea que solo hay un número finito de posibilidades con las cuales la molécula de ácido graso puede utilizar esta separación básica del exterior para su beneficio, antes de que sea necesario un cambio más complejo.
Hacia la artesanía generativa. Caso de estudio del oficio artesanal del barniz de Pasto (Colombia) y la impresión 3D

Cuando Steven Johnson traslada el adyacente posible a la interpretación de ecosistema de innovación, encuentra que las innovaciones han evolucionado en la historia siguiendo el patrón propuesto por Kauffman en los sistemas biológicos (Johnson 2011). Para Johnson, los límites de las ideas se conquistan solo para ser ampliadas. En otras palabras, el espacio de las posibilidades se expande cada vez que una idea se lleva al mundo real. El adyacente posible en un ecosistema de innovación, es el conjunto de novedades aún no generadas, pero que pueden llegar a implementarse en un único gradiente de reacción inmediata entre los componentes del sistema. Desde esta perspectiva, a continuación, se aborda la experimentación realizada con un grupo de artesanos del oficio artesanal del barniz de Pasto (Colombia) y la impresión 3D, con la intención de identificar los escenarios de un posible adyacente de este ecosistema artesanal.

\section{Contextualizando el barniz de Pasto}

El barniz de Pasto es un oficio artesanal característico del sur de Colombia, que en el año 2020 ha sido declarado Patrimonio Cultural Inmaterial de la Humanidad (UNESCO, 2020), el cual consiste en decorar objetos de madera a partir de una película delgada que se obtiene de la resina vegetal de los cogollos de un arbusto llamado mopa-mopa (Elaeagia pastoensis L.E. Mora). Este componente vegetal se transforma en películas flexibles que pueden recortarse con facilidad y adherirse de manera natural a objeto de diversas formas. Los registros sobre el uso de la técnica datan de épocas prehispánicas, donde era utilizada para recubrir e impermeabilizar vasos cónicos de madera llamados queros en los últimos años del Imperio inca (Barrera y Quiñones 2006:66). Sin embargo, fue en la época colonial, especialmente a finales del siglo xVIII, con la creación de las cofradías, donde la técnica se transformó en arte híbrido y se convirtió en un importante sector económico de manufactura preindustrial que se mantuvo hasta mediados del siglo xx (Gomezjurado 2014).

Aunque, en la actualidad, el barniz de Pasto goza de un reconocimiento internacional, la realidad es que este oficio artesanal presenta un relativo agotamiento creativo y un evidente envejecimiento generacional desde la primera década del siglo xxI (Insuasty 2019). A pesar de las múltiples experimentaciones llevadas a cabo por diferentes entidades nacionales y regionales, no ha sido posible repotencializar este oficio, en gran medida porque muchas de estas iniciativas se han fundamentado en propuestas con una clara orientación hacia el mercado, donde el artesano es pensado como mano de obra cualificada para reproducir objetos globales o locales. Con este marco contextual, el presente estudio realizó una investigación sobre el posible impacto creativo que el uso de la impresión 3D podría tener en el oficio artesanal del barniz 


\section{artnodes}

https://artnodes.uoc.edu

de Pasto, siguiendo el enfoque de posible adyacente, y bajo la concepción de que este tipo de artesanía tiene un carácter híbrido que evoluciona junto a sus artilugios tecnológicos con el paso del tiempo.

\section{Metodología de trabajo}

El procedimiento metodológico llevado a cabo se centró en la implementación de un conjunto de prototipos que sirvieran como objetos epistémicos donde fuera posible validar, de manera exploratoria, un conjunto de hipótesis investigativas (Boserman 2019). Este enfoque requiere que, además de la materialización de la idea por medio del prototipo, se pueda contextualizar la experiencia a través de un relato que contenga la visión de los actores al interactuar con el artefacto (Bleecker 2009; Kirby 2010). A continuación, se detallan estos aspectos.

\section{Implementación del prototipo}

Se determinó realizar un jarrón en impresión 3D al que se pudiera parametrizar tres criterios de diseño para su elaboración (altura del objeto, número de lados de la base y ángulo de rotación del cuerpo principal), con la finalidad de que el producto resultante tuviera la menor intervención humana en su diseño y se pudieran analizar los paradigmas creativos alrededor de los algoritmos de modelación morfológica de productos. Para este proceso, se utilizó Grasshopper, que es un plug-in de programación visual que corre dentro de la aplicación CAD, Rhinoceros 3D, donde se pudieron obtener más de cuatro mil geometrías de superficie a partir de la interacción de las variables parametrizadas en el componente Galápagos. El total de estas geometrías se introdujeron en un controlador para que, de manera aleatoria, se pudiera seleccionar, con un intervalo cada 200 milisegundos, una de las geometrías parametrizadas. La experimentación con el algoritmo se puede apreciar en la siguiente imagen.

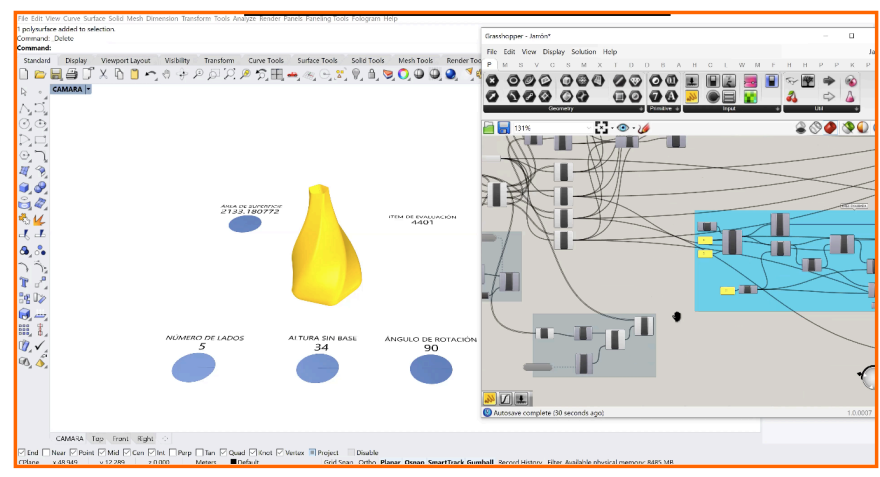

Imagen 1. Proceso de parametrización de los objetos contenedores
Hacia la artesanía generativa. Caso de estudio del oficio artesanal del barniz de Pasto (Colombia) y la impresión 3D

\section{Contextualización de la experiencia}

Seleccionada la geometría aleatoria, se imprimieron un total de seis jarrones en material PLA (Polylactic Acid), en una impresora 3D MakerGear M2 con la intención de que tres reconocidos maestros artesanos del barniz de Pasto, Germán Obando, Jesús Ceballos y Gilberto Granja, pudieran decorar estas piezas libremente con la técnica del barniz y tener un primer acercamiento a los procesos y las herramientas de fabricación digital. Finalizado este proceso de decorado, se solicitó a los artesanos expresar sus opiniones sobre la experiencia realizada, por medio de una entrevista no estructurada cuya principal intención fue identificar escenarios de posibles adyacentes entre el oficio artesanal y la fabricación digital. Los resultados obtenidos con la experiencia artesanal se pueden apreciar en la imagen adjunta.

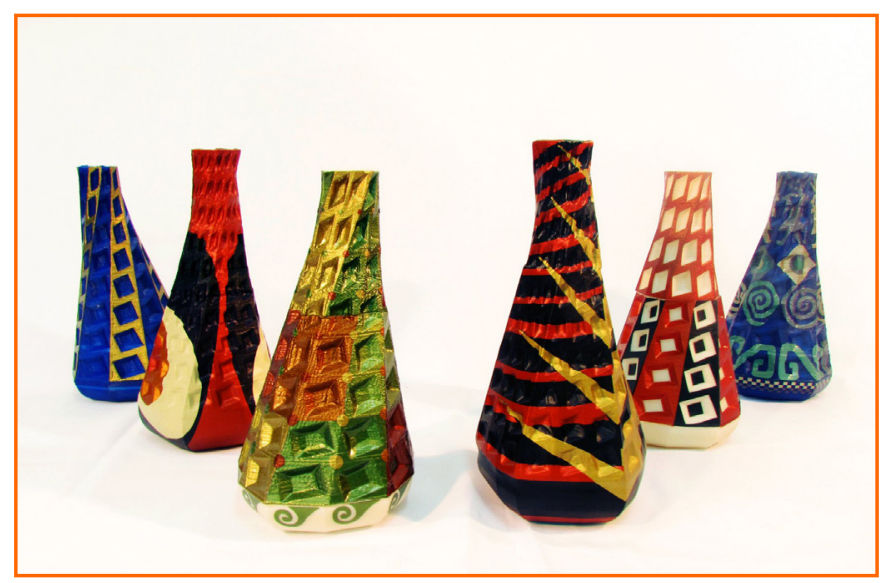

Imagen 2. Artefactos de impresión 3D decorados con la técnica del barniz de Pasto

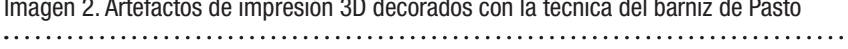

\section{Resultados y discusión: hacia la artesanía generativa}

Este ejercicio de experimentación obtuvo un total de 4.402 geometrías de superficie a partir de la parametrización de los componentes de diseño propuestos para el objeto contenedor, seis jarrones impresos en PLA y tres experiencias de decorado con barniz de pasto realizadas por reconocidos maestros del oficio artesanal. A continuación, se analizan por separado los resultados obtenidos en la implementación del prototipo y en la contextualización de la experiencia.

\section{Análisis sobre el prototipo}

En términos generales, el proceso de manufactura llevado a cabo en la experimentación permitió generar un conjunto de geometrías donde el ejercicio creativo no estuvo centrado en la morfología del objeto, sino en su algoritmo de construcción. Este enfoque de «estética 


\section{artnodes}

https://artnodes.uoc.edu

algorítmica», que no es otra cosa que el andamiaje lógico en el que se formula un nuevo tipo de lenguaje estético, ya ha sido abordado desde el arte generativo con el uso de los computadores como herramientas creativas (Cardoso 2009). De hecho, Jon McCormack y otros (McCormack, Bown, Dorin, McCabe, Monro y Whitelaw 2014) se cuestionan si este tipo de arte puede llegar a convertirse en una herramienta de arte comercial, y si más bien la práctica generativa deba entenderse como una forma de oficio artesanal. Este nuevo tipo de artesanía híbrida experimental, acompañada de procesos de fabricación digital y herramientas de parametrización, se conformaría como el primer gradiente de posible adyacente al que la artesanía gremial inevitablemente se desplegará, como ya parece estar ocurriendo en la actualidad. Basta con poner como ejemplo el caso de los proyectos de la firma mexicana dela0 Design Studio o las propuestas de mobiliario del diseñador Matthias Pliessnig, donde categorías como artesanía urbana o neoartesanía dejan de ser representativas para estos casos.

Por otra parte, el proceso de decorado de las piezas 3D buscaba determinar si la resina natural del barniz, de alto valor cultural para toda una región, mantenía sus características de adherencia, impermeabilidad y color al utilizarse en un polímero de amplio uso como es el PLA. Los resultados muestran claramente su viabilidad como «contenedor del oficio», donde el material utilizado puede jugar un papel determinante en la presentación visual y táctil del artefacto final, pues la impresión 3D posibilita la experimentación con diversos materiales para impresión como TPU flexible o filamentos lúdicos fluorescentes, metálicos o conductivos.

En este aspecto, el adyacente posible se configura alrededor de un tipo de artesanía experimental, muy cercana al objeto único de la artesanía preindustrial y al artefacto artístico. Aquí es donde cobran importancia los laboratorios de fabricación (FabLabs) como espacios de divulgación de conocimiento y producción creativa. Según la ley de Lass, el crecimiento de los FabLabs es de carácter exponencial, al igual que la ley de Moore sobre capacidad de procesamiento en los microprocesadores (Gershenfeld, Gershenfeld y Cutcher-Gershenfeld 2017:18). Esto representa un crecimiento distribuido de producción global y la formación de clusters creativos de ámbito local, como ha ocurrido con la reproducción del protector facial contra la COVID-19 de la empresa PRUSA 3D, el cual se estima que ha sido descargado más de medio millón de veces en todo el mundo por diversas comunidades de trabajo.

\section{Análisis sobre la experiencia}

De las entrevistas con los artesanos, se han extraído un conjunto de cuatro experiencias importantes que se detallan a continuación.

a) Mayor libertad morfológica. Para el maestro Jesús Ceballos, el diseño de formas a partir de procesos de parametrización permite
Hacia la artesanía generativa. Caso de estudio del oficio artesanal del barniz de Pasto (Colombia) y la impresión 3D

una mayor «posibilidad para vestir objetos» con la técnica del barniz. Es decir, para este artesano, la diversidad morfológica dada por el algoritmo de la máquina amplía de manera evidente la variedad de los contenedores formales donde se deposita el oficio artesanal. El barniz de Pasto, como artesanía híbrida, ha utilizado como contenedores del oficio objetos de madera, principalmente manufacturados en talla y torno. Sin embargo, esto no significa que este tipo de material deba ser el único en el cual se expresa el carácter simbólico del quehacer artesanal. De hecho, el barniz en su uso prehispánico, parece haberse empleado para decorar otros materiales como el oro y la plata (López 2012). Conocedor de este aspecto, la atención del maestro Ceballos parece orientarse a imaginar un escenario de mayor grado de libertad morfológica ofrecida por proceso de parametrización del objeto.

b) Mayor libertad de manufactura. Para los maestros Granja y Obando, el principal interés resultante de este ejercicio, se centró en la posibilidad de optimizar los costos de distribución del producto en el mercado. Para ellos, el bajo peso del material utilizado (PLA) ofrece «mayores ganancias por producto elaborado», según el maestro Granja, y una «mayor facilidad de transporte por peso cuando el producto se vende a otras partes del mundo», según el maestro Obando. Este enfoque pragmático de la artesanía gremial centrada en el mercado global confirma la existencia de una producción artesanal mecanizada con intervención de la mano de obra experta de la región, interesada en especializarse aún más para mantener un mercado cautivo. Es decir, los maestros parecen encontrar en la fabricación digital la posibilidad de diseñar localmente y producir de manera global, con lo cual perciben un escenario posible con mayor libertad de manufactura para bienes de nicho.

c) Mayor autonomía creativa. Otro aspecto importante encontrado ha sido el de los «objetos imposibles». Los maestros Granja y Ceballos hallaron en la tecnología de impresión 3D una oportunidad para materializar aquellos objetos que no han podido elaborar por limitaciones en las técnicas de manufactura de piezas torneadas en madera y los costos de las elaboradas en talla. Granja, por ejemplo, comenta que «ahora puedo imprimir mis propios diseños», mientras que Ceballos habla de imprimir una «forma compleja para decorar que nunca me han podido elaborar». Esta facilidad de materialización, lejos de negar el arte del barniz, parece darle un atractivo basado en el disfrute creativo y personal del artista-artesano. Los maestros mencionados identifican, entonces, un escenario de libertad autonómica orientada en la expresión simbólica. Sobre este aspecto, hay que tener en cuenta que, al hablar del derecho de autonomía artesanal, no se hace referencia a una cuestión étnica, sino a la «facultad de tomar desiciones atinentes a la regulación, interpretación y el sentido de conocimiento artesanal» (Osorio Pérez y Barrera Jurado, 2013). Vale la pena señalar aquí la gran subordinación que los artesanos del barniz han tenido durante los últimos cuarenta años sobre el tipo de diseño y objetos a elaborar (Insuasty, 2019). Este proceso de imposición realizado por expertos que desean representar al 0tro 


\section{artnodes}

https://artnodes.uoc.edu

desde lo diferente, con la intensión de categorizar lo diferente desde la normalidad occidental, se convierte, según Oswaldo Granda (2008), en un ejercicio de suplantación y antropofagia cultural denominado síndrome de Montaigne, y que, en el caso de la artesanía, se ve reflejado en limitantes creativas sobre diseños y objetos por criterios de mercado y moda.

d) Limitaciones de la experiencia. En términos generales, los tres maestros artesanos encuentran que la principal desventaja identificada en la experiencia con la fabricación digital se halla en la falta de competencias suficientes para utilizar y apropiar adecuadamente estos dispositivos tecnológicos. Aunque no verbalizan de manera directa este inconveniente, lo cierto es que, a pesar de su interés, todos expresan una inicial resistencia tecnológica a la fabricación digital, por ejemplo, cuando el maestro Ceballos denomina la impresora 3D como «máquina compleja». En este aspecto se concentra el principal reto de la artesanía generativa, pues como posible adyacente puede convertirse fácilmente en un componente de brecha digital. Se espera que este tipo de estudios exploratorios abonen el camino para que se implementen más investigaciones y trabajos de alfabetización tecnológica con los colectivos artesanales que preservan un determinado acervo cultural.

\section{Conclusiones}

Utilizando el enfoque del posible adyacente, se pudo analizar, a través de un ejercicio de experimentación con el oficio artesanal del barniz de Pasto y la impresión 3D, las implicaciones de la fabricación digital en un sistema artesanal híbrido. El proceso de experimentación identificó, con ayuda de tres maestros artesanos, una serie de escenarios posibles donde la fabricación digital podría impactar el devenir de este oficio artesanal. Con los resultados obtenidos, se ha propuesto que el conjunto de estos escenarios caracteriza el posible adyacente de la artesanía actual. Denominada artesanía generativa por tener un enfoque hacia una «estética algorítmica», este posible adyacente se compone de un primer escenario que hace referencia a la libertad morfológica, un segundo escenario que hace referencia a la libertad de manufactura y un tercer escenario que hace referencia a la libertad creativa.

Cada uno de estos entornos constituyen una oportunidad para el sistema artesanal, siempre y cuando existan procesos de alfabetización en fabricación digital dirigidos a artesanos, como ya se ha establecido en otros proyectos similares (González 2018). En caso contrario, su resistencia tecnológica podría convertir la artesanía generativa en una amenaza al acervo cultural. De igual manera, es importante dejar claro el aspecto exploratorio de esta investigación, la cual se constituye en un primer acercamiento a este posible adyacente. Para finalizar, es evidente la necesidad de mayores estudios sobre este tema, especialmente sobre caracterizar en detalle los
Hacia la artesanía generativa. Caso de estudio del oficio artesanal del barniz de Pasto (Colombia) y la impresión 3D

escenarios aquí propuestos, así como ahondar en metodologías para alfabetización en fabricación digital para artesanos, y estudios sobre el papel del diseñador-creador en las nuevas estéticas algorítmicas.

\section{Agradecimientos}

Los autores quieren expresar un agradecimiento especial a los maestros artesanos del barniz de Pasto Germán Obando, Jesús Ceballos y Gilberto Granja. De igual manera, agradecen al equipo del FabLab de la Universidad de Nariño, conformado por el profesor Harold Bonilla y Ios estudiantes Javier Perez Nastar, Andrés Caicedo, Carlos Castro y Martín Patiño. Por último, desean reconocer el trabajo realizado por el profesor de arquitectura Andrés Alexander Caicedo en el algoritmo de modelación paramétrica.

\section{Referencias bibliográficas}

Acha, Juan. 1979. Arte y sociedad. Latinoamérica. Sistemas de producción. México, D.F: Fondo de Cultura Económica.

Andersen, Hanne Voldborg, y Kati Pitkänen. 2019. «Empowering Educators by Developing Professional Practice in Digital Fabrication and Design Thinking». International Journal of ChildComputer Interaction 21: 1-16. D0I: https://doi.org/10.1016/j. ijcci.2019.03.001.

Anderson, Chris. 2012. Makers: The New Industrial Revolution. New York: Crown Business.

Atkinson, Paul. 2004. «Post-industrial manufacturing systems : the impact of emerging technologies on design, craft and engineering processes». En Challenging craft : International Conference, 8-10 septiembre, 2004, editado por Gordon Burnett. Aberdeen, Scotland: Gray's School of Art, Robert Gordon University. http:// shura.shu.ac.uk/8662/.

Barrera, Gloria, y Cielo Quiñones. 2006. Conspirando con los artesanos. Bogotá: Pontificia Universidad Javeriana.

Bleecker, Julian. 2009. «Design Fiction: A Short Essay on Design, Science, Fact and Fiction». Near Future Laboratory 97.

Boserman, Carla 2019. «Rescuing Epistemic Objects from Speculative Design». Revista Diseña 14: 118-37. D0I: https://doi.org/10.7764/ disena.14.118-137.

Cardoso, Daniel. 2009. «Generative Craft A Brief Critical Inquiry into Design Automation and Design Automata». Panorama 1: 288-290. Dorfles, Gillo. 1973. El diseño industrial y su estética. Barcelona: Labor. Freitag, Vanessa. 2014. «Entre arte y artesanía: elementos para pensar el oficio artesanal en la actualidad». El Artista 11: 129-143.

García Canclini, Néstor. 1990. Culturas Híbridas. México, D.F: Grijalbo.

García Canclini, Néstor. 1987. «Ni folklórico ni masivo: ¿qué es lo popular?» Diálogos de Comunicación 17: 8. 


\section{artnodes}

https://artnodes.uoc.edu

Gaviria, Enrique. 2002. El liberalismo y la insurrección de los artesanos contra el librecambio. Primeras manifestaciones socialistas en Colombia. Bogotá: Universidad Jorge Tadeo Lozano.

Gershenfeld, Neil. 2012. «How to Make Almost Anything». Foreign Affairs 91(6): 43-57.

Gershenfeld, Neil, Alan Gershenfeld, y Joel Cutcher-Gershenfeld. 2017. Designing Reality: How to Survive and Thrive in the Third Digital Revolution. New York: Basic Book.

Gomezjurado, Álvaro. 2014. El barniz de Pasto: Testimonio del mestizaje cultural en el sur occidente colombiano, 1542-1777. Medellín: Universidad Nacional de Colombia.

González, Walter. 2018. El impacto tecnológico en la artesanía peruana. FAB LAB. Lima, Perú: Universidad Nacional de Ingeniería.

Granda, Oswaldo. 2008. Estética y Otredad en el Caribe. Barranquilla, Colombia: Travesía.

Insuasty, Germán Arturo. 2019. «Valoración del Patrimonio Cultural asociado con el Barniz de Pasto Mopa-Mopa, a partir de la participación ciudadana.» Tesis Maestría en Diseño para la Innovación Social, Universidad de Nariño. DOl: https://doi.org/10.22267/madis.4.

Johnson, Steven. 2011. Las buenas ideas: Una historia natural de la innovación. Madrid: Turner Publicaciones.

Kauffman, Stuart. 1996. «Investigations on the character of autonomous agents and the worlds they mutually create.» Investigations. Santa Fe: Santa Fe Institute. https://www.santafe.edu/research/ results/working-papers/investigations.

Kirby, David. 2010. «The Future Is Now: Diegetic Prototypes and the Role of Popular Films in Generating Real-World Technological Development». Social Studies of Science 40(1): 41-70. DOI: https:// doi.org/10.1177/0306312709338325.

López, María del Pilar. 2012. «Oriente en el Nuevo Reino de Granada. Influencias y presencias en los objetos artísticos. El caso del
Hacia la artesanía generativa. Caso de estudio del oficio artesanal del barniz de Pasto (Colombia) y la impresión 3D

arte del barniz de Pasto.» En Ásia en América. VI Jornadas Internacionales de Arte, Historia y Cultura Colonial, 86-117. Bogotá: Ministerio de Cultura, Museo Colonial, Museo Santa Clara.

Marshall, Justin. 2002. "Craft and Technology». En Craft in the TwentyFirst Century. 15-17 noviembre, 2002. Edinburgh: School of Art.

McCormack, Jon, Oliver Bown, Alan Dorin, Jonathan McCabe, Gordon Monro, y Mitchell Whitelaw. 2014. «Ten Questions Concerning Generative Computer Art». Leonardo 47(2): 135-41. D0I: https:// doi.org/10.1162/LEON_a_00533.

Merchán, M J., P. Merchán, S. Salamanca, E. Pérez y T. Nogales, 2019. «Digital Fabrication of Cultural Heritage Artwork Replicas. In the Search for Resilience and Socio-Cultural Commitment». Digital Applications in Archaeology and Cultural Heritage 15: 1-11. DOI: https://doi.org/10.1016/j.daach.2019.e00125.

Osorio Pérez, Flor Edilma, y Gloria Stella Barrera Jurado. 2013. «Por los caminos de la autonomía comunitaria: debates y experiencias desde la autonomía artesanal kamsá». Tabula Rasa 19: 245-65. DOI: https://doi.org/10.25058/20112742.162.

Piore, Michael J., y Charles F. Sabel. 1999. La segunda ruptura industrial. Madrid: Alianza.

Scrase, Timothy. 2003. «Precarious Production: Globalization and Artisan Labour in the Third World». Third World Quarterly 24(3): 449-461. D0I: https://doi.org/10.1080/0143659032000084401

UNESCO. 2020. «The fifteenth session of the Intergovernmental Committee for the Safeguarding of the Intangible Cultural Heritage. Traditional knowledge and techniques associated with Pasto Varnish mopa-mopa of Putumayo and Nariño». Draft Decision: 15.COM 8.a.1 LHE/20/15.COM/INF.1. Paris, 10 December 2020. https://ich.unesco.org/en/15com

Vega, Daniel. 2012. «El aprendizaje de la Artesanía y su reproducción social en Colombia». Educación y Territorio 2(1): 89-112. 


\section{artnodes}

\section{CV}

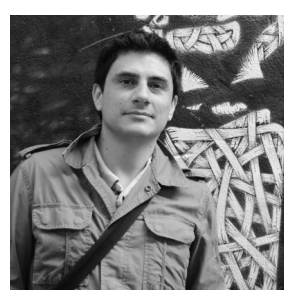

\section{Carlos Córdoba-Cely}

Universidad de Nariño, Colombia

cordobacely@udenar.edu.co

Doctor en Ingeniería Multimedia de la Universidad Politécnica de Cataluña, es diseñador industrial de la Universidad Javeriana, y en la actualidad es director de la Maestría en Diseño para la Innovación Social de la Universidad de Nariño, Colombia. Ha participado en diferentes proyectos de investigación centrados en fabricación digital e innovación social, y obtuvo en 2010 una mención de honor en el Seoul Design Competition 2010, realizado en Corea del Sur, con su proyecto Silla Pallarés. Entre los años 2016 y 2017 se desempeñó como secretario TIC de la Gobernación de Nariño, donde implementó el primer gobierno abierto regional de Colombia y lideró el desarrollo de diferentes plataformas de datos abiertos, por lo que logró en el año 2017 los premios INDIGO a la mejor innovación digital para un estado transparente del Ministerio TIC de Colombia.

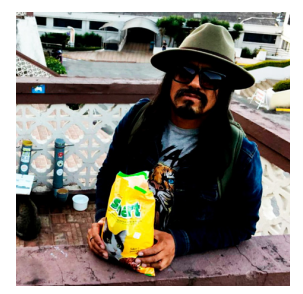

\section{Arturo de la Cruz Escobar}

Universidad de Nariño, Colombia

arturodelacruz@udenar.edu.co

Magíster en Diseño y Creación Interactiva de la Universidad de Caldas, diseñador gráfico-ingeniero de sistemas de la Universidad de Nariño, docente de la Maestría en Diseño e Innovación Social y de la especialización en Pedagogía de la Creatividad de la Universidad de Nariño, docente de pregrado de Diseño Gráfico de la Universidad de Nariño y en la Universidad CESMAG. Integrante del FabLab de la Universidad de Nariño. Dicta las asignaturas de animación, artefactos interactivos electrónicos en Arduino, fundamentos de programación para diseñadores y fundamentos semióticos para diseñadores. 